\title{
Stem cells from human exfoliated deciduous teeth differentiate into functional hepatocyte-like cells by herbal medicine
}

\author{
Wen-Ta Su* and Xiao-Wei Chen \\ Department of Chemical Engineering and Biotechnology, National Taipei University of Technology, 1 \\ Sec. 3, Chung-Hsiao E. Rd, Taipei 10608, Taiwan
}

\begin{abstract}
Stem cells from human exfoliated deciduous teeth (SHEDs) are mesenchymal stem cells isolated from the exfoliated human deciduous incisor that can differentiate into a many cell types. In this study, we evaluated the effect of liquorice or angelica extracts on the hepatic differentiation potential of SHEDs cells. SHEDs cells cultured in medium containing liquorice extracts were analyzed for 1) changes in cellular morphology, 2) changes in hepatic gene expression, AFP (Alpha-fetoprotein) and ALB (Albumin), and 3) albumin secretion and urea synthesis activity. Our data show that the hepatic differentiation potential of SHEDs cells is enhanced by the presence of liquorice or angelica extracts in the culture medium. Our findings present new therapeutic possibilities for liver damage repair.
\end{abstract}

Keywords: SHEDs, liquorice, angelica, differentiation, hepatocytes

\section{Introduction}

Liver is a primary internal organ involved in almost all physiological activities directly or indirectly. Chronic hepatic failure usually requires organ transplantation, which is limited by donor shortage and high mortality rates. Hepatocyte transplantation is simpler and less expensive than whole liver transplantation. However, this form of therapy is limited by the low availability of mature functional hepatocytes. The therapeutic potential of stem cells in liver diseases and regeneration has been confirmed in vitro and in vivo [1,2]. These stem cells can differentiate into hepatocyte-like cells capable of performing routine liver functions such as albumin production and urea metabolism [3]. Adult stem cell is an appropriate cell source with plasticity and has no ethical issues attached. Therefore, SHEDs cells are an appropriate choice for hepatic cell therapy [4,5].

SHEDs cells may serve as a suitable cellular source in tissue engineering [6]. SHEDs cells offer the advantage of rapid proliferation, easy isolation, and vast differentiation, making them a valuable resource of stem cells for the regeneration and repair of craniofacial defects, tooth loss, and bones [7].

Herbal medicine has been used to treat or prevent liver disease in Eastern countries. More recently,

\footnotetext{
${ }^{*}$ Corresponding author: Wen-Ta Su, Department of Chemical Engineering and Biotechnology, National Taipei University of Technology, 1 Sec. 3, Chung-Hsiao E. Rd, Taipei 10608, Taiwan. Tel.: +886-2-27712171 ext. 2554; Fax: +886-2-27519204; E-mail: f10549@ntut.edu.tw
} 
standardized herbs have been prescribed for patients with liver dysfunctions. The extracts of liquorice and angelica are used in traditional Chinese medicine and have many medicinal functions. This study examines the effect of liquorice or angelica extracts on the hepatic differentiation potential of SHEDs cells.

\section{Materials and methods}

\subsection{Isolated process of stem cells and routine culture}

SHEDs cells were first isolated from three different individuals according to the procedure outlined by Miura et al. [8]. Cells were then suspended in aMEM medium (Gibco) supplemented with $10 \%$ FBS, $20.0 \mathrm{ng} / \mathrm{mL}$ of BFGF and $100.0 \mu \mathrm{M}$ Ascorbic acid for cellular proliferation. Cell surface marker measurements were determined using flow cytometry (BD-FACS, Taiwan) and analyzed using the WinMDI software.

\subsection{Cellular morphology observation}

SHEDs cells seeded on tissue culture petri dishes were cultured in the presence of liquorice (1 $\mathrm{mg} / \mathrm{mL})$ or angelica $(0.1 \mathrm{mg} / \mathrm{mL})$ extracts in the growth medium. Control cells received no herbal supplement. Changes in cellular morphology were examined using fluorescence microscopy (Zeiss Axioplan 2; Axiovision software).

\subsection{Immunocytochemistry and functional evaluation by ELISA performing}

SHEDs cells grown on petri dishes were fixed with $4.0 \%$ formaldehyde solution for $0.5 \mathrm{~h}$, followed by washes with Phosphate-Buffered Saline (PBS). Cells were then incubated with anti-AFP (1:100) or anti-ALB (1:100) antibodies for $1 \mathrm{~h}$, washed three times with PBS, followed by the addition of secondary antibody Alexa Fluor (1:100) for $1 \mathrm{~h}$.

As a measure of hepatocyte function, albumin secretion and urea synthesis were determined using the Albumin assay kit (DIUR-250) and Urea assay kit (DIUR-500) respectively. Glycogen accumulation was determined by Periodic acid-Schiff staining.

Table 1

The sequences of primers used for the real time PCR

\begin{tabular}{ll}
\hline Gene name & primer sequences \\
\hline AFP & F: 5'- CACTGCTGCAACTCTTCGTA-3' \\
\hline R: 5'- CTTTGGACCCTCTTCTGTGA-3' \\
\hline ALB & F: 5'- TCTTCGTCTCCGGCTCTG-3' \\
\hline R: 5'- CTGGCAACTTCATGCAAAT-3' \\
\hline FAPDH 5 '-ATGAGAAGTATGACAACAGCC-3' \\
\hline
\end{tabular}




\subsection{Gene expression by RT-PCR}

Total RNA from SHEDs cells were extracted using TRIzol. $500 \mathrm{ng}$ of RNA was used per cDNA synthesis reaction using SuperScript ${ }^{\circledR}$ III Reverse Transcriptase kit according to manufacturer's instructions (Invitrogen). RT-PCR was performed using target genes and GAPDH as a control. The sequences of PCR primers are listed in Table 1.

\section{Results and discussion}

\subsection{Characterization of SHED cells}

As determined by flow cytometry, SHEDs cells expressed markers of mesenchymal stem cells, such as CD90 (99.3\%), CD73 (99.2\%), OCT-4 (96.4\%), CD105 (44.5\%) and SSEA4 (64.2\%). However, isolated cells do not express CD34 or CD45. These results suggest that like mesenchymal stem cells, SHEDs cells have the ability to differentiate into multiple cell lineages, making them attractive candidates for tissue engineering [9]. The cellular morphology after 21 days of herbal induction is shown in Figure 1.

Immunocytochemistry and functional evaluation In order to verify the differentiation potential of SHEDs cells into liver cells [10], SHEDs cells were incubated in culture medium containing extracts of liquorice or angelica, followed by examination of hepatic markers. According to Figure 2, cultured

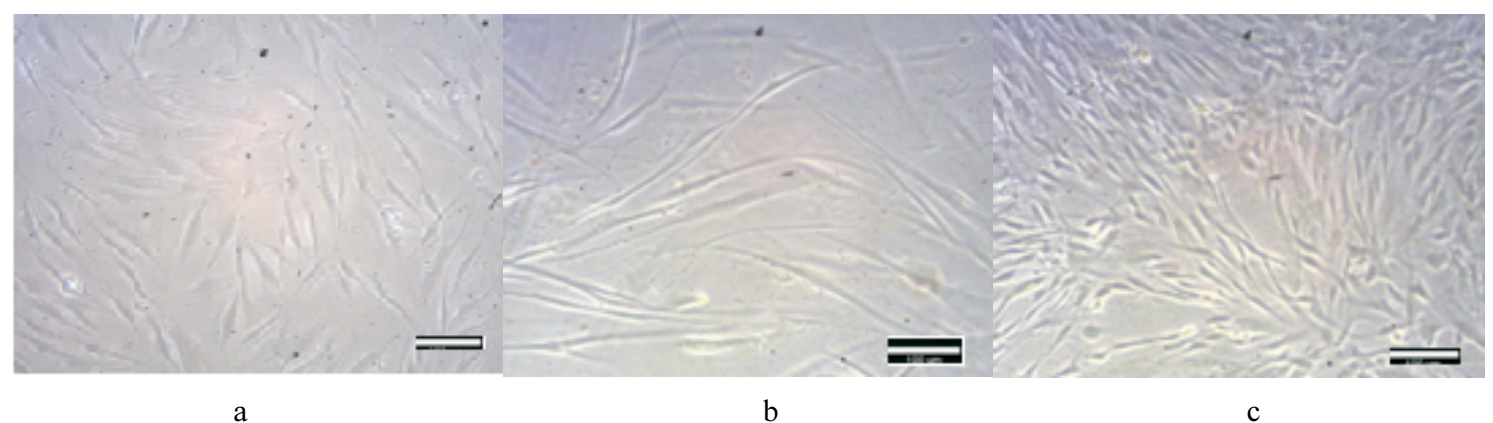

Fig. 1. Cellular morphology of non-induced (a) and induced SHEDs cells after 21 days culture (b: liquorice inducing, c: angelica inducing). Scale bar $100 \mathrm{um}$.

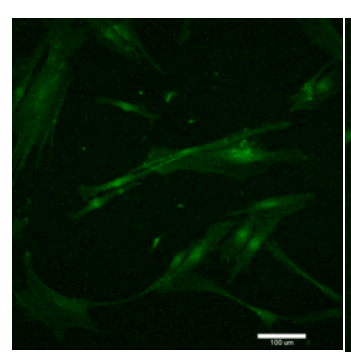

a

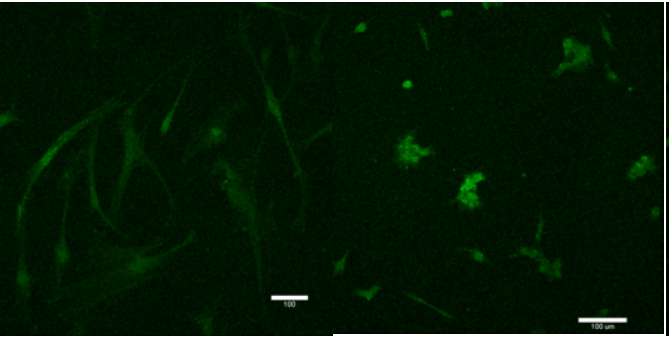

$\mathrm{b}$

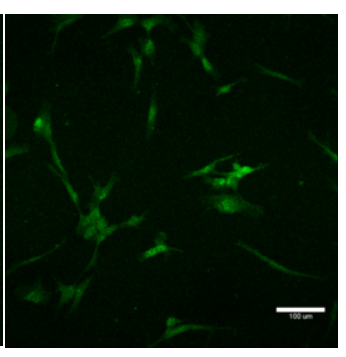

d

Fig. 2. The AFP (a) and ALB (b) shown by liquorice inducing, and AFP (c) and ALB (d) represented by angelica inducing. Scale bar $100 \mathrm{um}$. 
SHEDs cells show positive staining for hepatic markers such as AFP and ALB after 21 days in culture. These results show that in the presence of liquorice or angelica, SHEDs cells can readily differentiate into liver cells in vitro.
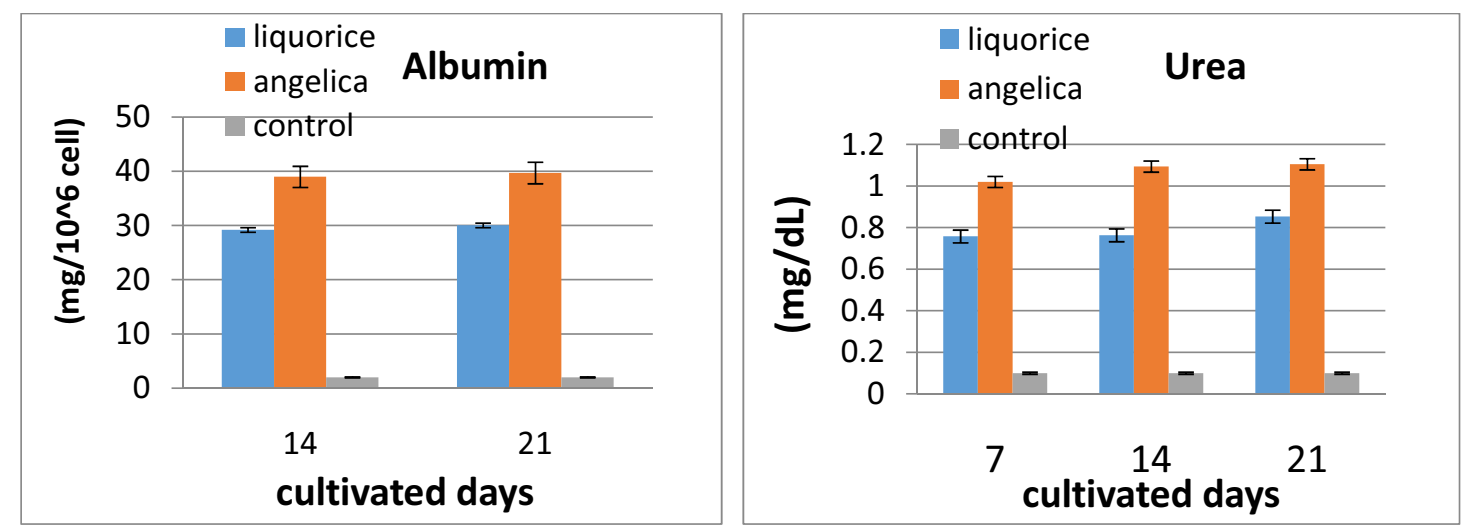

Fig. 3. The urea synthesis capacity and albumin secretion activity of differentiated cells. Values are expressed as mean \pm SD $(\mathrm{n}=3),{ }^{*} \mathrm{p}<0.05$

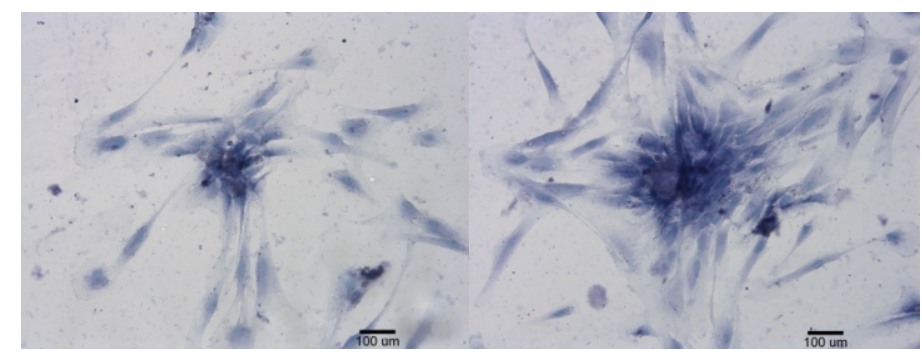

Fig. 4. The staining of glycogen accumulation by liquorice inducing (left) and angelica inducing (right). Scale bar $100 \mathrm{um}$.
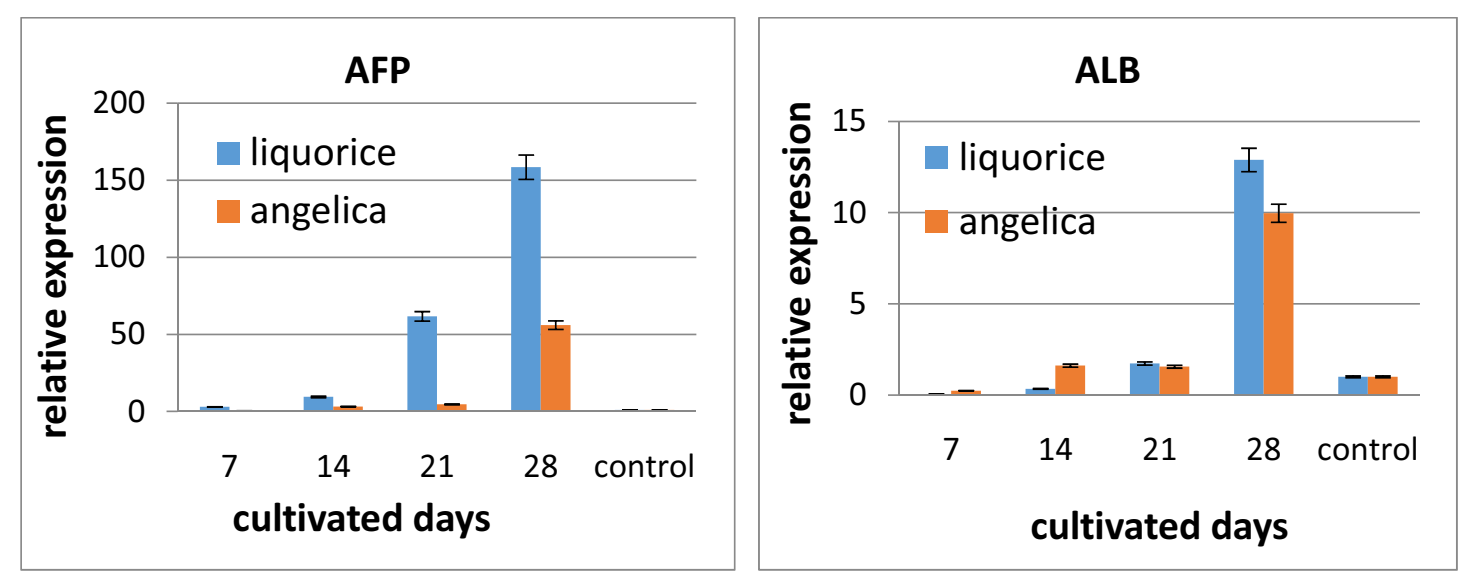

Fig. 5. The gene expression of AFP and ALB after liquorice and angelica inducing. Values are expressed as mean $\pm \operatorname{SD}(n=3)$, $* \mathrm{p}<0.05$ 
The albumin secretion and urea synthesis activity of differentiated cells are shown in Figure 3. Angelica extracts induced both higher albumin secretion and urea synthesis than liquorice extracts at various culturing times. Figure 4 show herbal induced glycogen accumulation

\subsection{Gene expression}

Herbal induction of AFP and ALB gene expression was observed in Figure 5 [11]. Notably, liquorice induction was higher than that of angelica for all the time points tested. Based on these results, both the extracts of liquorice and angelica can induce hepatic differentiation from SHEDs cells. While liquorice extracts induced higher expression of AFP and ALB, angelica was better at promoting albumin secretion and urea synthesis.

\section{Conclusion}

This study confirmed that herbal medicines such as liquorice and angelica can induce the hepatic differentiation of SHEDs cells, which may provide a cellular based therapy for hepatic failure.

\section{Acknowledgement}

The authors would like to thank the National Science Council of Taiwan for financial support under contract No. NSC 102-2622-E-027-025-CC3.

\section{Reference}

[1] D.C. Hay, D. Zhao, J. Fletcher, Z.A. Hewitt, D. McLean, A. Urruticoechea-Uriguen, J.R. Black, C. Elcombe, J.A. Ross, R. Wolf and W. Cui, Efficient differentiation of hepatocytes from human embryonic stem cells exhibiting markers recapitulating liver development in vivo, Stem Cells 26 (2008), 894-902.

[2] M. Vosough, M. Moslem, B. Pournasr and H Baharvand, Cell based therapeutics for liver disorders. Br. Med Bull. 100 (2011), 157-172.

[3] Z. Zhang, J. Liu, Y. Liu, Z, Li, W.Q. Gao and Z. He, Generation, characterization and potential therapeutic applications of mature and functional hepatocytes from stem cells, J. Cell Physiol. 228 (2013), 298-305.

[4] W.T. Su, P.S. Wu, C.S. Ko and T.Y. Huang, Osteogenic differentiation and mineralization of human exfoliated deciduous teeth stem cells on modified chitosan scaffold. Mater. Sci. Eng. C 41(2014) 152-160.

[5] C.B. Sangan and D.Tosh, Hepatic progenitor cells, Cell Tissue Res. 342 (2010), 131-137.

[6] P.D. Telles, M.A.A.M. Machado and V.T. Sakai, Pulp tissue from primary teeth: New source of stem cells, J. Appl. Oral. Sci. 19 (2011), 189-194.

[7] Y. Nishino, Y. Yamada and K. Ebisawa, Stem cells from exfoliated deciduous teeth (SHED) enhance wound healing and possibility of novel cell therapy, Chemotherapy 13 (2011), 598-605.

[8] M. Miura, S. Gronthos, M. Zhao, B. Lu, L.W. Fisher, P.G. Robey and S. Shi, SHED: Stem cells from human exfoliated deciduous teeth, Proceedings of the National Academy of Sciences of the United States of America 100 (2003), 5807-5812.

[9] C.Y.Lin, L.T. Li and W.T. Su, Study of subcellular dynamics on cell-substrate interactions by live cell imaging, J. Biomed. Mater. Res. Part A 102 (2014), 1149-1154.

[10] W.T. Su, Y.F. Liao, T.W. Wu, B.J. Wang and Y.Y. Shih, Microgrooved patterns enhanced PC12 cell growth, orientation, neurite elongation, and neuritogenesis, J. Biomed. Mater. Res. Part A 101 (2013), 185-194.

[11] N. Kim, H. Kim, I. Jung, Y. Kim, D. Kim and T.M. Han, Expression profiles of miRNAs in human embryonic stem cells during hepatocyte differentiation, Hepatol. Res. 41 (2011), 170-183. 